\title{
LÉGISLATION
}

\section{Les Procès engagés par ou contre les Concessionnaires. Question des Tribunaux compétents.}

\author{
Par Paul BoUgAULT, Avocat à la Cour d'Appel de Lyon.
}

\begin{abstract}
Un procès est souvent compliqué par lui-même; il est peut-être plus compliqué encore par le doute dans lequet se trouvent les demandeurs au sujet du tribunal à choisir. L'étude que l'on va lire résume les principes généraux.
\end{abstract}

Définition de la concession d'énergie électrique. - On a donné de très nombreuses définitions du mot "concession "; cette multiplicité provient de ce fait que ce contrat ayant avec d'autres, beaucoup de points comniuns, sans cependant se confondre avec eux, on a été amené à forger des définitions pour les besoins de la cause, afin de rendre plus particulièrement saillante l'opposition que l'on voulait faire avec d'autres contrats. L'idée première qui se dégage du mot "concession" est celle d'un "abandon" fait par une autorité, agissant comme propriétaire d'une chose ou d'un droit, au profit d'une autre personne. Sans parler de la concession perpétuelle ou temporaire de la place qu'une commune accorde à une faridle pour la sépulture de ses membres, dans un lieu public et municipal appelé cimetière, on peut citer : la concession des rivages de la mer pour l'établissement de bains publics, la concession autrefois donnée à titre perpétuel d'une mine dé houille ou de minerais, la concession de l'énergie hydrauLique visée par la loi du 16 octobre 1919 ; l'autorité concedante, soit qu'elle se considère comme propriétaire, des l'origine, des biens concédés (rivages de la mer) soit qu'elle ait à accomplir des formalités pour faire tomber une propriété préexistante, comme en ce qui concerne le tretonds, livre ce bien à l'exploitation faite par un tiers, la réglemente et réserve certains avantages pécuniaires ou autres au profit d'une collectivité dont elle représente légalement les intérêts.

Si l'on définit (1) la concession de travaux publics de

(1) Nous donnons ane définition courante susceptible d'ètrè retenue dans ses clétails essentiels par les praticiens qui nous lisent et même par les Tribunaux auministratiis. Si l'on voulait recourir à une veritable définition de jurisconsulte, il faudrait prendre celle de M. Hauriou, précis de droit administratif, page 754: "Une opération dans laquelle une concession temporaire des droits de puissance publique nécessaires à la construction d'un ouvrage public et son exploitation, sous forme de service public, pour le comple d'une administration publique, étant instituee d'une façon réglementaire, les conséquences financières de cette institution ainsi que les conclitions de la construction et de l'exploi tation de l'ouvrage sont regleses, entre l'administration et le concessionnaire, par un contrat passé avec cahier des charges et tari maximum. "C'est la seule qui contienne, en une phrase, la double indication de la siluation réglementaire et du contrat administratif ; la situation réglementaire signifie que la concession doit d'abord être orientée dans le sens de l'amélioration, même par de nouvelles réglementations, le public ne devant pas souffrir de la substitution de la concession à la régie ; le contrat administratif signifie que des conditions financières seront stipulées pour assurer la rémunération de l'exploitant, sans faire obstacle $\mathfrak{a}$ cle nouvelles dispositions réglementaires. la façon suivante : " l'opération par laquelle l'administration compétente accorde, en le réglementant, le droit de maintenir des ouvrages sur ou sous le domaine public, pendant un temps déterminé, moyennant certains avantages stipulés, tant au profit des intérêts collectifs que cette administration représente, qu'en vue de la rémunération de l'exploitant ", la distribution de l'énergie électrique rentre incontestablement dans la catégorie des concessions de ce genre. L'idée qui donne tout particulié rement à une occupation le caractère définitif de la concession se trouve dans les termes suivants : "Le droit contractuel de maintenir ${ }^{1}$ ) des ouvrages sur ou dans le domaine public. - Pour certaides occupations, le contrat n'existe pas ; dans d'autres cas, on ne voit pas l'ouvrage implanté dans le sol public ; ainsi, l'absence de contrat se révèle dans la permission de voirie qui comporte cependant une incorporation d'ouvrages au sol public. D'autre part, bien qu'il y ait contrat, il n'y a pas marché de travaux publics dans les conventions de services publics ; par exemple, le domaine public n'est pas occupé dans le sens matériel " d'emprise" par un ouvrage : le service d'autobus ou d'omnibus en est un exemple frappant et

(1) Nous pourrions même dire "entretenir " un ouvrage place dans le sol public par un service public ; car, comme le dit le commissaire du gouvernement, Pichat (atfaire Thérond, 4 mars 1910), dans ses conclusions rapportées dans Dalloz, 1912. 3. 57, la jurisprudence est tellement extensive que le simple entretien d'ouvrages incorporés à un sol public sulfit pour faire attribuer à tout contrat le caractere de travail public; ainsi, dans l'affaire Permann contre la Ville d'Armentières, l'arrêt du 27 juillet 1906 (Dalloz, 1908. 3., page 16), décide qu'un contrat ayant pour objet la garde d'un cimetière relève pour le tout du Tribunal administratif par le seul fait qu'il attribue au gardien l'exécution de travaux d'entretien général du cimetiěre, ledit lieu étant une dépendance du domaine communal affectée à un service public. On ne saurait mieux préciser que Laferrière, Juridiction administrative, page 609 , t. 2 : "Lorsqu'un contrat est mixte, il n'est pas nécessaire quele marché de travaux publics domine; il suffit qu'il y apparaisse, même sous une forme très atténuée pour que la compétence du Conseil de Préfecture en résulte. La jurisprudence a peutêtre ainsi donné à la loi de pluviose, an 8 , une extension que n'avaient pas prévue ses auteurs, mais elle n'en doit pas moins ctre tenue pour acquise. "Laferrière cite comme exemples les marchés de distribution d'eau et de gaz, du balayage et du net toiement des voies publiques, du service des prisons, des entreprises de pompes funèbres. Voir aussi pour les marchés de four nitures et d'installation de cloches, Conseil d'Etat, 5 aont 1014 et les renvois (D. P. 1906.3.48. Affaire Escoubet contre fabrique de l'église de Mirande). Cette jurisprudence était applicable dans l'espèce, où le concessionmaire, pendant tout le temps de sa concession, était tenu de l'exécution de travaux publics. 
se distingue essentiellement du tramway : Ies denx opérations comportent bien le droit de faire stationner des voitures sur un endroit déterminé de la voirie, nais, en plus, la seconde entraîne le droit et l'obligation d'y incorporer des rails.

Dans le marché de fournitures, l'autorité contractante se propose évidemment de servir ou de protéger des intérêts collectifs (achat de combustibles pour chauffage des salles communes, de pavés pour les rues et les routes), mais, d'une part, le contrat ne comporte (pas plus que le service public) aucune occupation du sol, et, d'autre part, la prestation qui doit être faite à un jour donné n'embrasse pas une série de services à rendre pendant un temps plus on moins long.

Négliger cette distinction, c'est s'exposer à saisir, en cas de difficulté, un Tribunal radicalement incompétent (').

En principe, le contentieux des marchés de fourniture est le Tribunal de droit commun ; c'est ainsi que les fournitures stipulées par une commune donnent lieu à des litiges à porter devant le Tribunal Civil. Il n'en est différemment que pour les marchés de fournitures de l'Ftat; ils sont régis par le décret du 11 juin 1806, qui donne compétence au Conseil d'Elat pour statuer sur les décisions prises par les ministres au sujet d'une discussion resultant d'un marché $\left({ }^{2}\right)$.

(1) Il a longtemps semblé, à beaucoup de techniciens, que la définition exacte d'un contrat administratif devait être reléguée dans le domaine des questions purement juridiques, c'est-à-dire constituant une querelle d'école. Des événements importants sont venus en démontrer le caractère essentiellement pratique.

a) La loi sur les loyers du 9 mars 1918 n'est applicable qu'aux baux immobiliers : quand une commune a donné à bail un casino, il faut, pour savoir s'il y a bail ou service public des eaux dont le casino ne serait que l'accessoire, interpréter le contrat : la Cour de Toulouse, dans un arrêt du 10 février 1919 (Gaz. des Trib., 31 août 1919 et Communication du Contențieux, No 349 , janvier 1920), reconnaît le caractère principal du contrat dans la concession des eaux, le service public dominant le bail accessoire du casino.

b) La loi Failliot (21 janvier 1918) n'est pas applicable aux baux immobiliers, mais senlement aux contrats commerciaux. La Cour d'Appel de Grenoble, le 4 novembre 1919 (Com. Cont. No 366 , année 1920 , page 361 ), décide que le contrat, paŕ lequel la Société Thermale d'Uriage a donné à son concessionnaire Buisson tous les immeubles qu'elle possédait, est dominé par le caractère de bail immobilier et échappe à la résiliation possible d'après la loi Failliot.

c) La diminution de jouissance, en vertu de l'article 1722 du Code Civil entraîne, au profit du preneur le bénéfice d'une diminution de prix. La Cour de Paris, dans un arrêt du 3 mars 1917, reconnaît le caractère de bail immobilier à la concèssion du Casino de Biarritz; estimant que, par la suppression des jeux pendant la guerre, Ies locataires Boulant et Catelain ont subi une diminution certaine dans la possibilité de jouir pleinement des lieux loués, la Cour accorde une réduction de loyer (Com. Cont., No 347 , année 1919, page 205). Déjà la Cour de Paris, dans un arrêt du 8 mars 1911, confirmé par un arrêt de la Chambre Givile du 18 juin 1912, avait décidé que la concession d'un terrain par une Ville, à la charge d'y construire un casino, n'avait en aucune façon le caractère de travail public (Voir ces arrêts et le rapport du Conseiller Feuilloley dans la Com. Cont., année 1912, page 56, Communication No 201).

(2) Il peut exister une certaine difficulté pour la distinction entre le marché de fournitures et le marché de travaux publics, quand le premier comporte un certain élément de travail, comme, par exemple, des conditions de montage, d'assemblage des fournitures livrées. II v aura lien d'analvser le montrat et de lui donner
Le contentieux des travaux publics est dévolu en premier ressort au Conseil de Préfecture et sur appel au Conseil d'Etat en vertu de l'article 4 de la loi de Pluviose, an 8 qui est ainsi conçu : "Le Conseil de Préfecture pronon" cera... sur les difficultés qui pourront s'élever entre les " entrepreneurs de trabaux publics et l'administration, "concernant le sens et l'exécution de leurs marchés ".

Le contentieux d'un service public général, ou simplement communal, appartient directement au Conseil d'Etat, en vertu de l'article 9 de la loi du 24 mai 1872 qui attribue à cette juridiction : "compétence souveraine sur le recours en matière contentieuse administrativen. Dans une affaire Terrier que le Conseil d'Etat a jugé le 6 février 1903 (voir les conclusions du Commissaire du Gouvernement dans Dalloz, 1904. 3. 65), il s'agissait d'une discussion au sujet d'un paiement de primes sur le crédit ouvert par le Conseil général sur le budget départemental pour la destruction des vipères. Ce n'était ni un travail public, ni un marché de fournitures. M. Romieu a revendiqué pour le Conseil d'Etat le droit de compétence absolue pour connaître d'un pareil litige. is Tout ce qui concerne " l'organisation et le tonctionnement des services publics is proprement dits généraux ou locaux, soit que l'admi"nistration agisse par voie de contrat, soit qu'elle procèdé par voie d'autorité, constitue une opération admi" nistrative qui est, par sa nature même, du domaine de "Ja juridiction administrative, au point de vne des litiges " de toutes sortes auxquels elle peut donner lien, ce quic "l'on peut encore exprimer ainsi : toutes les actions entre "les personnes publiques et les tiers ou entre ces person"nes publiques elles-mêmes fondées sur l'exécution, "l'inexécution ou la mauvaise exécution d'un service "public sont de la compétence administrative et relè"vent, à défaut d'un texte spécial, du Conseil d'Etat, "juge de droit commun du contentieux de l'adminis"tration publique, générale ou locale." C'est la même solution qui a été adoptée dans l'affaire Thérond (1 avril 1910, Dalloz 1912, 3. 57) où l'on voit la Ville de Montpellier concéder le service de l'enlèvement des bêtes mortes trouvées sur la voie publique, en y ajoutant le privilège de les prendre aussi chez les particuliers, contrat communal de louage de scrvice qui, pour employer l'expression de $M$. Pichat, revêt la forme d'une concession ; mais comme celle-ci ne serait susceptible d'etre exécutée que si le Maire, par un arrêté illégal (Conseil d'Etat,

sa définition exacte d'après l'élément prédominant. Sur ce point M. Blum, Commissaire du Gouvernement, a donné, dans l’affaire Société de Granit des Vosges contre Ville de Lille (24 juillet 1912 . Com. Cont., No 198 , année 1912 , page 311 ), des conclusions remarquables de nettetc. Appelant contrat " administratif " le marché de travaux publics, et contrat "ordinaire" le marché de fournitures, il faut valoir, en s'appuyant sur différents arrêts (I)épartement de Maine et Loire, 11 novembre 1910 ; Lamy, 26 janvier. 1912 Agence Nationale d'Affichage, 10 mai 1912), qu'en cas de doute, il faut reconnaître le caractere de contrat administratif. à celui qui reste teinté et influencé par le service public pour leque] il est conclu; pour qu'un marché communal de fournitures devienne un contrat de travaux publics, il faut qu'il ćtablisse des rapports précis du lournisseur ou de ses agents, soit avec la commune, soit avec le public ; il faut qu'l associe le fournisseur dans une mesure quelconque à la gestion du service. Mais lorsque le marché, au contraire, n'établit pas entre la commune et le contractant des rapports différents de ceux pouvant exister entre un particulier quelcongue et un marchand quelconque, la commune a agi comme un particulier , la competence est celle des Tribunaux ordinaires. 
18 janvier 1901. Affaire Chaigneau, Dalloz 1902. 3. 50 et Ch. Crim., 22 juillet 1899 , affaire Chaigneau contre ministère public, Dalloz 1901. 1. 482), imposait aux habitants de livrer les bêtes à l'équarisseur public, elle doit être déclarée nulle ( ${ }^{1}$ ). Pour le dire, une seule juridiction est compétente : le Conseil d'Etat:

Difficultés entre la commune et le concessionnaire. Compétence. - La Cour de Cassation, comme le Conseil d'Etat attribue le caractère de contrat de travail public à toute concession de gaz et d'électricité ; il en est ainsi

(1) Monsieur le Commissaire du Gouvernement a fait l'historique de la transformation d'une jurisprudence qui avait débuté d'une manière toute différente : le Conseil d'Etat ne devait d'abord connaitre que les recours dirigés contre des actes d'autorité entachés d'illégalité. Le Tribunal des Conflits a affirmé la compétence exclusive et absolue de la juridiction administrative qui ne peut être que celle du Conseil d'Etat) pour connaitre d'abord la question de la responsabilité de l'Etat dérivant du fait de ses agents ; dans l'affaire Blanco, il s'agissait de faire condamner l'Etat, assigné devant le Tribunal Judiciaire en même temps que ses ouvriers et comme responsable de leur fait, parce que dans la Manufacture des Tabacs de Bordeaux, en manouvrant un wagonnet, ils avaient blessé une petite fille. La décision du Tribunal des Conflits, du 1ér février 1873, décide que l'action intentée par le demandeur a pour objet de faire déclarer l'Etat civilement responsable, par application des articles 1382,1383 , 1384 du Code Civil, de l'imprudence de ses ouvriers, que cette responsabilité n'est ni générale, ni absolue, qu'elle a ses règles spéciales suivant les besoins du service; qu'aux termes des lois des 16-24 août 1790, titre 2 , article 13 et 16 Frudictor an 3 , la séparation des pouvoirs veut que l'autorité administrative soit seule compétente (Voir Trib. des Confl., $1^{\text {er }}$ février 1873, Blanco, Dalloz, 1873. 3. 20). Même solution quand le département est attaqué en raison de la responsabilité d'un Préfet, à l'occasion d'un incendie allumé à une meule de paille par un aliéné dangereux qui s'était évadé d'un asile départemental. Il est évident que le Préfet ne peut être responsable d'une mauvaise organisation. d'un service tout entier ; personnellement, il n'est pas chargé de surveiller les aliénés (Trib. des Confl., 29 février 1908, affaire Feutry contre Préfet de l'Oise, Dalloz 1908. 3. 49) ; même solution encore quand une commune est actionnée en raison de la faute de service commise par ses préposés qui avaient, pendant un jour d'orage, ouvert les vannes du canal de Marseille (de Fonscolombe contre Ville de Marseille, Trib. des Confl., 11 avril 1908 , Dalloz 1908. $3,67)$, et, enfin; solution identique dans une affaire basée sur le même acte, mais dirigée contre l'Association Syndicale du canal de Grignac par Jouillé (Trib. des Confl., 26 mai 1908. Jouillé contre Ass. Synd, de Grignac. Dalloz 1908. 3. 67). Ladite association est prise dans cette hypothèse comme un établissement public, c'est-à-dire un service public spécial personnifié (Voir. sur ce point, Hanrion, page 771).

De cette orientation cantonnée, tout d'abord, par la jurisprudence, dans le domaine de la responsabilité, est née, en matière contractuelle, une orientation identique, dessaisissant la juridiction judiciaire au profit du Conseil d'Etat. Sans doute, il n'y a eu aucun changement pour les contrats de l'Etat et des colonies, car la juridiction administrative reçoit une pleine compétence indiquée à des textes spéciaux (décret du 10, juin 1806, article 13). Mais, en ce qui concerne les communes, on peut citer plusieurs arrêts : il ne faut pas attacher une trop grande importance à l'arrêt commune de Saissac contre Melliés du 28 mars 1888 (Dalloz 1889. 3. 53) ; en présence d'une fourniture de pétrole et d'un louage de service consistant dans l'allumage et l'extinction des reverbères, le Conseil d'Etat a déclaré que le Conseil de Prélecture avait eu tort de se déclarer compétent; ce qui n'stait pas douteux; seulement, il n'a pas donné d'indication au sujet de la caractéristique principale du contrat : devait-on le considérer comme un marché de fournitures (dans ce cas le Tribunal judiciaire aurait été compétent exclusivement à tout autre) ou comme une concession de louage de service (avec compétence du Conseil d'Etat). La Jurisprudence s'est nettement affirmée dans l'arrêt précité Thérond, du 4 avril 1910, qui n'a visé qu'un louage de service, bien nettement caractérisé. depuis 1880 ; auparavant, ees deux juridictions faisaient une distinction entre deux éléments du contrat. La partie relative à l'installation des ouvrages dans la voirie étali seule considérée comme un travail public ; la partie rela. tive à la distribution du gaz était justiciable des tribu: naux de droit commun de même que les conditions finan. cières du contrat. Le Tribunal des Conflits a rompu ave cette tradition et affirmé la compétence exclusive du Conseil de Préfecture, pour juger entre le concédant et le concessionnaire, toutes les contestations (internrétation $\left({ }^{1}\right)$ ou exécution.

La décision du Tribunal des Conrlits du 16 décem bre 1876 , rapportée dans Dalloz 1877.3 .57 , avec une note très complète, était relative à une discussion entre la Ville de Lyon et la Compagnie du Gaz de la Guillotière, sur une clause du contrat prévoyant une participation éventuelle aux bénéfices de l'exploitation. Quatre ans après, la Cour de Cássation se ralliait à cette jurisprudence dans une atfaire oì la Compagnie, l'Union des Gaz, reprochait à la Ville de Nîmes d'avoir, en cours de contrit, surélevé ses tarifs d'octroi au préjudice de sa concessionnaire (arrêt du 2 mars 1880. Chambre Civile, Dalloz 1880. 1. 231). Le Conseil d'Etat admet depuis fort longtemps les mêmes principes ; on ne saurait citer un arrêt plus complet que celui du 14 juin 1912, affaire Mottet contre commune de Saint-Martin-Vésubie (Com. Cont. 1912, page 277, No 196) ; le Conseil d'Etat déclare : a) que Ie principe de la compétence administrative n'est en rien modifié du fait qu'un article du contrat attribue à la Municipalité le droit d'aequérir l'installation complète de l'éclairage électrique " moyennant un prix fixé à dire d'experts, dont un choisi pąr la Municipalité, et, en cas de désaccord, entre les deux, un tiers expert étant nommé par le Juge de Paix $" ; b$ ) que l'évaluation faite par ledit expert doit être considérée comme un renseignement susceptible d'être discuté par le Conseil de Préfecture; $c$ ) - mais que si, cependant, le Conseil estime qu'il doit recourir à une nesure d'instruction et de vérification, il n'est pas lié par ce fait qu'un expert a été nommé par le Juge de Paix qui était ineompétent, mais il lui incombe de nommer un autre expert, conformément à la loi du 22 juillet 1889 .

Une commune peut-elle intenter directement un procès contre'son concessionnaire pour soutenir les interêts de ses consommateurs qu'elle juge sacrifiés?

La question ne peut se résoudre que par une distinction.

Si une commune trouve, dans son cahier des charges, un article formel qui lui permette d'intervenir dans lilitérêt général des consommateurs, elle peut intenter au principal une action dont le but sera de contraindre le concessionnaire au respect du contrat. Un arrêt du 5 avril 1884 a décidé qu'elle était en droit de demander le respect d'un article de son traité, pour obtenir l'abaissement des tarifs dans certaines conditions-prévues (Conseil d'Etat, Affaire Compagnie Parisienne du Gaz contre Ville de Paris, 5 avril 1884 , Dalloz 1885. 5. 271). Mais la

(1) Il faut, évidemment, pour que le Tribunal administratif soit complètement saisi, qu'il existe entre la commune et le concessionnaire une difficulté réelle ; on ne saurait obtenir une consultation " du Conseil de Préfecture sans qu'elle soit annulee par le Conseil d'Etat (Conseil d'Etat : Ville d'Oran contre Compagnie Centrale du Gaz, 19 mars 1875. Dalloz 1875. 3. 107). 
question s'est présentée d'une facon toute spéciale, en ce qui concerne la rédaction des polices à imposer aux consommateurs. Une commune peut, si elle a joint à -son cahier des charges une police type, assigner directement son concessionnaire devant le Conseil de Préfecture et demander la modification de certains termes de ce type, pour le motif que, d'après ses prétentions au moins, ces termes sont contraires aux textes du traité : la recevabilité de son action et la compétence du Conseil de Préfecture proviennent de ce fait qu'il y a incorporation du modèle type au traité municipal, et que ce traité peut toujours, pour interprétation, être soumis par une commune au juge administratif (Voir arrêt du Conseil d'Etat du 4 décembre 1885 qui a reconnu l'action de la commune de Saint-Mandé recevable, Dalloz 1887. 3. 47).

Même si, dans le traité qui ne contient pas de modèle, il a été inséré un article rendant nécessaire l'approbation par le Maire de tout modèle à établir, la commune peut discuter les termes de la police avec son concessionnaire devant le Tribunal administratif. Mais elle ne peut refuser son approbation que si la police qui lui est proposée est contraire au cahier des charges ; et une commune qui, par erreur, prétendrait que le prix proposé par le concessionnaire est en contradiction avec le cahier des charges, et se refuserait par ce motif à approuver un projet, engagerait sa responsabilité ( 29 janvier 1875 , Ville de Chartres. Rec. Lebon, page 77 ).

Mais si la clause, sans être contraire au cahier des charges, est seulement non prévue, la commune n'a pas qualité pour s'y opposer, par exemple, pour demander qu'une Compagnie concessionnaire n'inscrive pas dans ses polices l'entretien du compteur à la charge de l'abonné, quand la fourniture au compteur n'est pas prévue au cahier des charges qui n'envisage que la fourniture à la jauge (Compagnie générale des Eaux contre commune de Clichy, 3 mars 1893. Dalloz 1894. 3. 42). Car la commune ne peut se considérer comme investie d'un mandat légal de ses habitants pour faire respecter leurs droits que s'il existe, entre elle et son concessionnaire, un lien direct que la juridiction administrative peut facilement saisir, par exemple un principe à faire maintenir tel qu'il est écrit dans le cahier, ou une violation formelle de ce contrat à empêcher. Si la clause que le concessionmaire discute avec les consommateurs n'est pas en opposition directe avec le cahier des charges, la commune ne peut pas faire juger la question : la simple omission dans un cahier, ou un systèmé non prévu au moment du contrat, ne pouvant être assimilés à une violation d'un texte administratif (Conseil d'Etat, commune de Neuilly ; Rec. Lebon, page $732,\left({ }^{1}\right)$.

(1) On sait qu'aux termes de Ja Ioi du 5 avril 1884, article 123 tout contribuable inscrit au rôle de la Commune a le droit d'exercer à ses frais et risqués, avec l'autorisation du. Conseil de Préfecture, les actions qu'il croit appartenir à la commune ou section et que celle-ci préalablement appelée à en délibérer a refusé ou négligé d'exercer ; la commune ou section de commune est mise en cause et la décision qui intervient a effet à cet égard. Par conséquent, quand un particulier croit que la commune a le droit d'exiger, d'après son cahier des charges, certains avantages pour les consommateurs, il est susceptible d'être tenté par la rapidité et la simplicité de la procédure administrative ; il ira plus promptement que s'il assignait le concessionnaire devant le Tribunal de Commerce, avec demande de renvoi au Tribunal administratif pour faire interpréter le cahier des charges. Mais il faut remarquer que le contribuable peut agir seulement s'il est démontré que l'action mise en ouvre appartiendrait bien à la commune; on ne
Conflits entre les consommateurs et les concessionnaires. - Ces conflits relèvent toujours de la juridiction judiciaire ou commerciale et il n'appartient jamais au Tribunal administratif de prononcer une condamnation au profit d'un particulier contre le concessionnaire ou inversement (Conseil d'Etat, 14 novembre 1879. Compagnie du Gaz d'Arles contre Puech, Dalloz, 1880. 3. 30).-Conseil d'Etat, 28 juillet 1916. Société du Gaz du Midi, contre Société Chapellerie Montilienne. Voir Communication du Comité du Contentieux de la Chambre Syndicale des Forces Hydrauliques, année 1918, page 97, No 319). Seulement, dans certains cas, les juges de droit commun ne pouvant interpréter un contrat administratif renvoient le plaideur devant le Conseil de Préfecture, pour solliciter l'interprétation nécessaire, et revenir ensuite devant eux, afin d'entendre la condamnation ou le rejet de la demande.

La seule difficulté est de reconnaître quels sont les cas où le Tribunal de droit commun peut statuer immédiatement, et quels sont les cas où il doit passer par cet échelon intermédiaire, c'est ce que nous allons essayer d'établir clairement :

10. Il tombe sous le sens, tout d'abord, que si le conflit est né d'une police à interpréter pour des causes n'intéressant en rien le mode de fourniture ou le paiement, la solution doit être donnée en dehors de toute immixtion du Tribunal administratif : par exemple, une police doit-elle ètre considérée comme faite par son signataire, pour lui seul ou pour son successeur? (').

Ne doit-on pas déclarer nulle, comme sans cause et viciée par la violence, une transaction passée entre un abonné et la Compagnie des Eaux de Paris, quand cette Compagnie, voulant à tout prix recouvrer les sommes à elle dues par le maître d'un lavoir public, a imposé au successeur de celui-ci, comme prix d'une police immédiate et indispensable au fonctionnement du lavoir, le paiement préalable d'une somme de 500 francs ? Il est évident qu'en répondant affirmativement par jugement du 16 décembre 1910 , le Tribunal de la Seine ne faisait que trancher une discussion purement privée (Voir le Journal Le Droit, du 27 décembre 1910, et Gaz. des Trib., 20 décembre 1910).

$2^{\circ}$ La mème décision doit être appliquée dans le cas où le consommateur demande à avoir tel ou tel tarif an lieu de tel ou tel autre; car il s'agit de déterminer la catégorie à laquelle il appartient : il en serait de mème si la qualité du courant fourni à ce consommateur était en discussion : le concessionnaire étant tenu évidemment de donner la qualité prescrite au cahier des charges, la question porte sur le point de savoir si la fourniture qui est faite, est conforme aux exigences du contrat initial. C'est évidemment

saurait, par exemple, admettre qu'un consommateur fit trancher par cette voie, en l'absence de tout désaccord entre la commune et le concessionnaire sur l'exécution du traité, la question de savoir s'il a le droit d'exiger un abonnement (Conseil d'Etat, Compagnie générale des Eaux contre Michel, 21 novembre 1890 . Dalloz 1892. 3. 50 )

(2) Voir Cassation, 14 avril 1885 (Dalloz $1886,1,30$; affaire Douine contre Société d'Eclairage au Gaz) ; Cassation, $6 \mathrm{le}$. vrier 1899 (Dalloz 1899.1 .280 , affaire Barrere-Lherbier), Gou de Lyon, 1er avril 1908 (Revue de l'Hyglene, année 1909 , page 69 affaire Compagnie des Eaux contre Habitants de Montchat)

"L'action intentée par un propriétaire contre la Compagnie " concessionnaire, afin de faire condamner celle-ci a abonner "directement ses localaires aux conditions des polices, est de la "compétence du Tribunal ordinaire ": tel est le sommaire de l'arrêt de la Cour de Lyon. 
un conflit dans lequel l'interprétation du cahier des charges n'a pas à être donnée ( ${ }^{1}$ ). Il a même été jugé par la Cour d'Amiens, le 16 février 1917 (Com. Cont., 1919, No 326), que si le cahier des charges dispense le concessionnaire, en cas de force majeure, de faire la fourniture, il n'y a pas lieu à renvoi, le Tribunal de Commerce étant compétent pour rechercher s'il n'y a pas eu, en fait, faute du distributeur.

$3^{\circ}$ Du reste, quelle que soit la téndance du litige, ancune difficulté ne se produit quand le consommateur demande au distributeur d'exécuter purement et simplement le cahier des charges qui est parfaitement clair. Il s'agit pour la juridiction judiciaire de faire une application pure et simple du texte administratif : ce qui est tonjours dans le pouvoir du juge, à la différence de l'interprétation de ce texte -Cassation, 29 avril 1885. Dalloz, 1886, 1. 239; affaire Valette, et Dalloz, supplément Vo Travaux Publics, No 1954).

Ainsi, un propriétaire allègue que le concessionnaire, par son cahier des charges, est tenu de faire une colonne montante quand le propriétaire lui assure au moins trois abonnés de trois ans ; une simple lecture du cahier suffit pour bien connaitre les obligations du concessionnaire, et les juges du Tribunal Civil savent lire : il n'y a lieu qu'à application et le juge la fait directement (Cassation, 11 janvier 1911, Mon. Jud., du 25 avril 1911, rejet d'un pourvoi contre un arrêt de la Cour de Paris, du 5 janvier 1909).

Mais il faut s'arrêter à ce point, et dès que l'interprétation, si minime qu'elle soit, est nécessaire (c'est-à-dire si le Tribunal peut avoir le plus léger doute sur la signification du cahier des charges), il doit surseoir à statuer et renvoyer les parties au- Tribunal administratif, jusqu'à ce que la solution ait été donnée par celui-ci $\left(^{2}\right)$. Bien des procès ont été perdus à la Cour de Cassation, parce que, pour gagner du temps, les parties plaidantes avaient poussé le Tribunal Civil à donner lui-même l'interprétation du cahier des charges. Ainsi, par exemple, la Cour de Paris avait cru pouvoir, en lisant le règlement de la distribution des eaux en vigueur dans la commune de Charenton, tirer de certains articles des conséquences qui n'y étaient pas écrites, mais qu'elle prétendait en faire découler par un simple raisonnement. Son arrêt du 24 décembre 1902 a été cassé par la Cour Suprême en date du 23 juin 1909 (Dalloz, 1910. 1. 23, affaire Compagnie des Eaux contre Berluque).

(1) Le prononcé de ce renvoi est nécessaire et les parties ne peuvent s'adresser directement elles-mêmes au Tribunal administratif qui, pour elles, n'est qu'un Tribunal d'exception : il y aurait donc impossibilité d'avoir une décision qui ne serait pas préalablement basée sur le renvoi du Tribunal judiciaire (Conseil de Préfecture de la Seine du 6 mai 1910 ; Gazette des Tribunaux du 27 juin 1910; (Communication du Contentieux de la Chambre Syndicale des Forces Hydrauliques, année 1910, page 345 , No 126).

(2) Cassation, 24 avril 1885 (Dalloz 1186. 1.239; affaire Valette, Pallud et Greck) ; Cassation, 6 mars 1901 (Dalloz 1902. 1. 251; alfaire Meyronet contre Air comprimé). Dans ces deux affaires, il a été donné des solutions intéressantes sur de nombreuses questions : les 'difficultés invoquées par le consommateur, tirées de ce tait que ce n'était pas la Société exploitante, mais son prédécesseur qui avait signé le contrat, ou bien que ce n'était pas l'ayant droit lui-même, mais son vendeur ; enfin, on lira certains détails de force majeure alléguées pour la cessation d'un abonnement de la responsabilité d'un vice de construction dans les canalisations établies.
Si la police à interpréter fait corps avec le contrat ad. ministratif, l'interprétation est, à plus forte raison, dé fendue an Tribunal Civil (Cassation 30 juillet 1908. Dal loz, 1908. 1. 453 ; affaire Compagnie des Eaux d'Oran contre Drougnon).

La même solution a été donnée par la Cour d'Appel de Toulouse dans le cas suivant : un abonné prétendait qu'en lui faisant signer sa police le concessionnaire avait violé ses dispositions de son cahier des charges. Et il y avait une certaine obscurité dans la fixation de la portée exacfé du contrat administratif : chacune des parties prétendait y voir ce que l'autre n'y voyait pas : la Cour a renvoye; devant le Tribunal administratif. Les Tribunaux judiciaires doivent même surseoir à statuer sur un litige entre la Compagnie concessionnaire et ses ouvriers an sujet de la détermination des salaires, si la contestation nécessite l'interprétation d'une clause obscure ou ambiguë du cahiel des charges. Cassation, 9 juillet 1913, Compagnie générale du Gaz de Bordeaux contre Bordel et autres (Com. Cont., année 1914, page 102).

Enfin, une discussion s'étant élevée à Auxerre pour savoir si le concessionnaire de l'éclairage et de la force était tenu de donner la force motrice aux usines pendant le jour seulement ou pendant la nuit et le jour, le Tribunal n'a condamné le concessionnaire à une fourniture permanente qu'après avoir reçu du Conseil d'Etat l'assurance que tel était bien le sens du cahier des charges (Voir arrêt du Conseil d'Etat du 5 janvier 1907, Dalloz, 1908. 3. 79. arrêt Soissons contre Compagnie Universelle d'Eclairage de Chauffage et de Force motrice, Com. Cont., F. H., No 20).

Les Tribunaux compétents pour juger les différends entre le concessionnaire et consommateur sont les suivants :

10 Le Tribunal de Commerce, qui doit être nécessairement saisi, si le litige est engagé entre le distributeur (qui est sûrement un commerçant) et un autre commerçant qü utilise le courant pour les besoins de son commerce. Le même Tribunal peut être saisi par un non-commerçant contre le distributeur ; le premier a le droit de choisir eette juridiction qui ne peut pas être récusée par le distributeur : un commerçant, en effet, ne saurait se plaindre d'être traduit devant son propre Tribunal.

$2^{\circ}$ Le Tribunal Civil, si le distributeur assigne un consommateur non commerçant, ce dernier ne pouvant être distrait de ses juges naturels; si c'est le consommateur qui assigne le distributeur, ce dernier, bien que commerçant ne peut récuser le Tribunal Civil, car il ne saurait, en effet, obliger un demandeur à recourir au Tribunal de Commere qui est un Tribunal d'exception. On peut consulter sur ce point le Répertoire pratique de Dalloz Vo compétence commerciale No 19 , et l'arrêt de la Chambre des Requêtes du 1 er juillet 1908, affaire Coulon contre Favaudon Dalloz, 1919. 1. 11; cet arrêt a non seulement reconnu que le Tribunal de droit commun est compétent pour juger un commerçant à la requête d'un non commerçant, mais que le second peut employer contre le premier les modes de preuve en usage au Tribunal de Commerce, bien que ce soit la juridiction de droit commun qui soit saisie.

$3^{\circ}$ Le juge de Paix, cette juridiction n'est compétente que si l'action engagée rentre par son taux et par sa nature dans le domaine de la compétence déterminée par la Ini du 12 juillet 1905 . 
Personne n'ignore que le juge de paix pour toutes actions pu rement personnelles ou mobilières est compétent pour statuer en dernier ressort jusqu'à une valeur de $300 \mathrm{fr}$. ; entre 300 et 600 francs, il ne juge qu'en premier ressort, c'est-à-dire que la partie perdante peut déférer le jugement au Tribunal Civil qui constitue le second degré (').

Dans les affaires qui nous occupent, la nature de l'action ne peut soulever aucune difficulté. Elle sera toujours ou personnelle, comme une demande de paiement de sommes arriérées ou mobilière comme une revendication d'objets mobiliers, par exemple un moteur, des lampes, etc.

Mais la détermination du taux du ressort présente des questions quelquefois compliquées ; par exemple, un consommateur demande au juge de paix d'abord de condamner le distributeur au paiement d'une somme de 150 francs, pour rupture intempestive de courant, et ensuite le rétablissement de ce courant, à peine d'une astreinte de $5 \mathrm{fr}$. par jour de retard pendant dix jours ; il semblerait au premier abord que cette demande dût être qualifiée en dernier ressort, puisque le demandeur lui-même n'en fait pas monter le chiffre même à 300 francs. Néanmoins, la Cour de

(1) La compétence du juge de paix en matière mobilière étant générale dans les limites du taux précité, il a le droit d'examiner tous les moyens qui sont opposés par le défendeur à la demande et doit statuer sur les questions d'interprétation, d'existence, de validitéet même sur le mérite d'offres réelles qui ont été pratiquées. Il ne peut y avoir de difficulté que lorsque le défendeur fait valoir un moyen qui n'a pas simplement pour but de repousser la demande, mais qui oblige le juge à statuer sur un droit propre au défendeur : il tombe sous le sens que l'ordre des compétences pourrait en être troublé : il y aura lieu alors d'appliquer les règles suivantes : si la demande principale et la demande reconventionnelle, chacune étant prise isolément, rentrent toutes les deux dans le cadre de la compétenco, le juge de paix peut statuer sur le tout en dernier ressort ; lorsque la demande reconventionnelle excède la compétence du juge de paix, celui-ci doit se dessaisir de cette demande, et il peut soit retenir le jugement de la demande principale, soit renvoyer sur le tout les parties a se pourvoir devant le Triburial de première instance sans préliminaire de conciliation; s'il garde à son Tribunal la demande principale, en renvoyant la demande reconventionnelle, son jugement ne peut être qu'en premier ressort (Répertoire pratique Vo Compétence Civile des Juges de Paix, numéros 46, 47 et suivants). Si cependant il s'agissait d'une demandee rconventionnelle en dommages-intérêts exclusivement basée sur la demande principale, la loi dispose que cette demande ne saurait rendre l'affaire susceptible d'appel, si le taux de la demande principale ne comporte pas le deuxième degré de juridiction.

Un arrêt extrêmement intéressant a été rendu le 10 décembre 1888 par la Chambre Civile, affaire Compagnie d'Assurances la Centrale contre Philjppe (Dalloz 1889. 1. 141) : il s'agissait d'une demande reconventionnelle au sujet de laquelle la Cour a admis que la valeur de la demande en résiliation d'une police d'assurance se détermine par la totalisation des primes qui seraient à payer jusqu'à l'expiration naturelle du contrat. cassation, dans un arrêt extrêmement intéressant, a déclarè qu'il n'appartient pas au demandeur, en chiffrant le montant des dommages-intérèts, de rendre "déterminée "la valeur d'une réclamation qui, par sa nature, est indéterminée.

Le demandeur réclamait en réalité deux choses bien distinctes : une somme très faible, comme réparation du préjudice causé pour le passé ; de ce chef aucune difficulté, car une somme est toujours une somme; il réclamait aussi la reprise de la fourniture du courant, dans un délai de dix jours, à peine de cinq francs par jour : or la restitution d'un courant n'a pas une valeur fixée ; elle est indépendante des dommages-intérêts dus pour la rupture, et de l'astreinte relative à la reprise, puisqu'à l'expiration du délai pendant lequel court l'astreinte, il faudra revenir devant le Tribumal pour être statué à fond. Le jugement, en vertu de.l'article 14 de la loi du 25 mai 1838 , ne pouvait être qu'en premier ressort. (Ch. civile, 18 janvier 1921). Cassation au profit de Magnau, propriétaire du Secteur d'éclairage de Bray-surSeine, par défaut contre Moreau d'un jugement din Tribunal civil de Provins du 12 avril 1916, qui avait déclaré irrecevable l'appel contre une décision du juge de paix de Bray-sur-Seine.

S'il est une question qui nous a été souvent poséc, c'est celle de la compétence du juge des référés que des personnes privées de courant, par un fait un peu brusque du distribubuteur, aimeraient à voir proclamer universellement : il est si commode de recourir à un magistrat qui juge rapidement et sans frais !

Malheureusement, s'il est vrai que le juge des référés ne peut être saisi qu'en cas d'urgence, cette condition " d'urgence "n'est pas suffisante ; il faut aussi que la solution que l'on demande au juge de donner sur l'heure, ne préjuge pas le fond ; larticle 809 du Code de Procédure Civile le dit expressément : « Les ordonnances sur référé ne feront aucun préjudice au principal. "C'est surtout dans le domaine du fait que la compétence du juge s'exerce (fermeture d'une excavation dangereuse, étayage d'un mur menaçant ruine, même nomination d'un.expert dans un litige qui serait de la compétence du Tribunal Civil). Il peut aussi s'avancer sur un terrain qui aurait été l'objet d'un contrat, mais à la condition que ce soit pour maintenir l'une des parties dans une prétention qui serait justifiée par un titre très sérieux. Au contraire, on considère généralement qu'il ne saurait dire si le courant a été coupé à tort sans faire lui-même l'interprétation du contrat tacite ou exprès qui est intervenu entre le distributeur et le consommateur. Ainsi, le Tribunal Civil de Briançon siégeant en référé s'est déclaré incompétent pour faire rétablir une fourniture de courant que le distributeur avait rompue sur le refus du consommateur de signer une police (Af. Jacob, 9 avril 1921. Bulletin des Usines Electriques, décembre 1920Août 1921. 